\title{
Lonergan, lector del \\ Ensayo para contribuir a una gramática del asentimiento
}

Lonergan, Reader of An Essay in Aid of a Grammar of Assent

\author{
Francisco Vicente Galán Vélez \\ Universidad Iberoamericana, México \\ francisco.galan@ibero.mx \\ Recibido: 17/11/2015 • Aceptado: 9/02/2016
}

\begin{abstract}
Resumen
Este artículo muestra la influencia que la lectura de la Gramática del Asentimiento ejerció en el libro de Bernard Lonergan Insight: estudio de la comprensión humana. Particularmente es notable a lo largo del análisis de la capacidad de juzgar la verdad de algunas afirmaciones. Se puede encontrar en cualquier ser humano lo que Newman llamó «sentido ilativo»; nuestra razonabilidad, así, resulta comparable a la prudencia - pero en el terreno cognoscitivo-. Lonergan señala que se trata de un procedimiento rudimentario e inevitable: no hay más fundamento del conocimiento que nuestro compromiso pragmático por querer conocer.
\end{abstract}

Palabras clave: asentimiento, juicio, razón, sentido ilativo, verdad.

\section{Abstract}

This article shows the influence that the reading of the Grammar of Assent exercises on Bernard Lonergan's book Insight: A Study of human understanding, especially in his analysis of our capacity to judge about the truth of a statement. Newman's illative sense is our reasonableness, which is similar to prudence, but in the cognitional domain, and we can find it in every human being. Lonergan says that it is a rudimentary and inevitable process, and that there is no more foundation for knowledge than our pragmatic engagement in our willing to know.

Keywords: assent, illative sense, judgment, reason, truth. 
Mi mentor y guía fundamental ha sido la Gramática del Asentimiento de John Henry Newman. La leí en mi tercer año de filosofía (por lo menos las partes analíticas) alrededor de unas cinco veces y encontré solución para mis problemas. No estaba del todo satisfecho con la filosofía que se me estaba enseñando, y encontré la presentación de Newman como algo que cuadraba con la manera en la que yo conocía las cosas. Fue desde ese núcleo que fui hacia diferentes autores.

Lonergan, 2004: $3889 .{ }^{1}$

Es bien conocido por los estudiosos de la obra de Lonergan que éste reconoció la Gramática del Asentimiento (GA) de Newman como su influencia fundamental y su guía filosófica. En este trabajo, analizo dicha influencia en la principal obra filosófica de Lonergan: Insight: Estudio sobre la comprensión humana. La influencia principal de la GA es lo que Newman llamó «sentido ilativo». ${ }^{2}$ Aunque, como trataré de mostrar, Lonergan recoge con mucho detalle el sentido ilativo en los capítulos centrales del Insight dedicados al análisis del papel del juicio en el conocimiento, ${ }^{3}$ lo curioso es que este concepto no aparece en el Insight, ni tampoco el nombre de Newman. Mi propósito no es únicamente histórico, sino que, como considero vigente

1 "My fundamental mentor and guide has been John Henry Newman's Grammar of Assent. I read that in my third year philosophy (at least the analytic parts) about five times and found solutions for my problems. I was not at all satisfied with the philosophy that was being taught and found Newman's presentation to be something that fitted in with the way I knew things. It was from that kernel that I went on to different authors". (La traducción de todas las citas en inglés es mía).

2 "La doctrina del sentido ilativo viene a ser la aportación característica y fundamental de la Grammar of Assent" (Athié, 2001: 79).

3 La influencia de Newman sobre Lonergan no se reduce a la GA. Philip A. Egan menciona que son dos las principales aportaciones de Newman a Lonergan: el sentido ilativo y el desarrollo histórico de la doctrina cristiana (2007: 1107). Aunque menor, en cuanto a que no es un tema que Lonergan trate mucho, estaría su influencia presente también en un tercer asunto: en el papel que la teología debe jugar en las universidades. 
la propuesta de Lonergan, trato de mostrar la pertinencia de la propuesta de Newman.

Una breve indicación del libro Insight

Bernard Lonergan (1904-1984), canadiense, jesuita, ${ }^{4}$ sobre todo fue un profesor de teología dogmática que hizo notables colaboraciones a la filosofía y a la economía. Es en torno a estos tres ejes disciplinares que gira su producción bibliográfica. Sus dos libros más importantes son el Insight, publicado en 1957, y Method in Theology (MT), publicado en 1971.

Es muy difícil hacer una presentación, aunque sea somera, del libro Insight, sin traicionar el espíritu pedagógico de la obra, que pretende guiar al lector a un aprendizaje real y no meramente nocional, de algo que le parece a Lonergan ser el centro de cualquier postura filosófica. Hay un núcleo de problemas que están entretejidos de modo esencial y, aunque solemos separarlos, son el corazón del misterio. Da lo mismo por dónde se empiece, siempre que se recorra todo el camino: "Al construir un barco o una filosofía, se tiene que recorrer todo el camino; un esfuerzo que por principio es incompleto equivale a un fracaso" (Lonergan, 1999: 15). Entre los problemas del conocimiento y los problemas de la realidad, entre epistemología y metafísica, hay una estrecha interpenetración. Insight quiere llevar a ese núcleo esencial, pero propone comenzar por algo anterior a la metafísica (filosofía antigua), y a la epistemología (filosofía moderna). En la introducción, se señala que el libro responde a dos preguntas que dan lugar a las dos grandes partes: "La

4 En la Compañía de Jesús, fue enviado al Heythrop College de Inglaterra para su formación en Humanidades (1926-1930). Fue durante aquellos años que, un poco decepcionado de la formación escolástica que recibía, la cual le parecía conceptualista, vino a encontrarse con la GA. Tuvo un profundo impacto en su modo de concebir el conocimiento. Hay varios escritos de Lonergan de este período que lo atestiguan; se les llama los Blandyke Papers, pues se publicaron en una revista de los estudiantes con ese nombre, y son: "La forma de la inferencia matemática" (escrito en enero de 1928), "El silogismo" (marzo de 1928), y el más importante, ya que es un ensayo directo sobre la GA, "Juicio verdadero y ciencia" (febrero de 1929). Todos ellos se encuentran en Lonergan, 2007. 
primera parte se refiere a la pregunta: ¿Qué sucede cuando estamos conociendo? La segunda parte, pasa a la pregunta: ¿Qué es lo que se conoce cuando eso sucede?" (Lonergan, 1999: 25). Lonergan propone, por motivos pedagógicos, que el lector se pregunte primero qué es lo que hace cuando conoce, y que después responda a las grandes preguntas sobre el fundamento del conocimiento y el universo del ser que se conoce cuando uno conoce.

Lonergan encontró un cierto conceptualismo en la formación filosófica que recibió de sus profesores suarecianos, a quienes no obstante se refiere elogiosamente por su preparación y responsabilidad. Muchos de quienes hemos vivido esto y hemos clamado por un sistema conceptual sólido, que resista a la más extravagante duda de cualquier escéptico, una y otra vez nos hemos topado con nuevas sutilezas que derrumban lo que se creía seguro. El fundamento no es ningún sistema conceptual. Ni siquiera la lógica misma. Del Insight y de la GA, podemos decir con Pierre Hadot (2006) que se tratan de textos que son una suerte de ejercicios espirituales. Tal vez no sobre el examen de todos los fundamentos. Pero sí sobre uno de ellos, que es muy importante respecto a otros: el auto examen del sujeto cognoscente. Insight da prioridad pedagógica a la explicitación de las capacidades que tenemos como sujetos y al reconocimiento de sus criterios normativos. Lonergan señala que fue Newman quien le hizo darse cuenta de la prioridad de lo existencial. ${ }^{5}$

Lonergan llamó en Insight «método empírico generalizado» y «método trascendental», en $M T$, a un esquema de operaciones intencionales que se pretende que existen y son ejercidas por todo ser humano y, por tanto, están presentes en todas las culturas y en todas las disciplinas cognoscitivas. Emplea la palabra «trascendental», por una parte, con cierto sentido kantiano: tales operaciones

5 De "Insight revisited": "I had become something of an existentialist from my study of Newman's A Grammar of Assent. (...) Insight was the fruit of all this. It enabled me to achieve in myself what since has been called Die antropologische Wende" (Lonergan, 1974: 276).

"Me había convertido en una especie de existencialista por mi estudio de la Gramática del Asentimiento de Newman. [...] Insight fue el fruto de todo ello. Me permitió lograr en mí mismo lo que ha sido llamado Die antropologische Wende (el giro antropológico)". 
son condición de posibilidad necesaria y universal del conocimiento; son a priori, pero no como un contenido cognoscitivo que de algún modo ya estuviera con anterioridad a la experiencia, sino en el sentido, ya presente en Aristóteles y santo Tomás, de que en su funcionamiento se orientan siempre hacia un objeto, o mejor dicho a un objetivo. Pero también son trascendentales en el sentido escolástico, en el que se aludía a las nociones «singularísimas», como el «ser»o la «unidad», que están más allá de las determinaciones categoriales y que, más que llamarles «universales», deberíamos llamarles «universalísimas», pues penetran y subyacen a todo contenido conceptual. ${ }^{6}$

Tales operaciones se ejercen de modo implícito en el ejercicio del proceso cognoscitivo de todos los seres humanos. El método empírico generalizado busca hacer explícito lo que todo mundo ya siempre ha conocido, por haberlo ejercido. Lonergan no pretende tener ninguna originalidad en su descubrimiento. Las operaciones ya han sido advertidas por grandes maestros del método empírico generalizado, entre los que sin duda debemos mencionar a Newman. Lonergan se atribuye el mérito de poner en una sola perspectiva lo que otros fueron tematizando. Las operaciones de este esquema tienen dos propiedades: son intencionales, ya que el sujeto se percata de algo, y son conscientes, ya que el sujeto se percata de sí mismo al realizarlas. Se pueden agrupar en cuatro niveles de consciencia o de intencionalidad, que son: 1) el nivel de experimentar: ver, oír, oler, tocar, gustar; 2) comprender: preguntar, imaginar, inteligir, concebir, formular; 3) juzgar si lo comprendido es correcto o no: deliberar, ordenar y ponderar la evidencia, juzgar; 4) planear y decidir sobre planes de acción. En el Insight, se explicitan los tres primeros niveles de consciencia por ser los que están involucrados en el conocimiento, de modo que si conocer es experimentar, entender y

6 Sin embargo, llamarle «método trascendental», se presta a confusiones. Por eso muchos lonerganeanos prefieren volver al nombre de «método empírico generalizado», el cual parece más cercano a la fenomenología de Husserl que a Kant. La cita de Newman como su mentor y guía, la hizo Lonergan para deslindarse de que se le colocara dentro del tomismo trascendental, como un discípulo de Maréchal. Si bien, Lonergan menciona que conoció a Maréchal gracias a un compañero griego jesuita, y que lo leyó con agrado, de ninguna manera considera que ejerciera el influjo que ejerció la $G A$. 
juzgar, conocer el conocimiento será duplicar la estructura, es decir experimentar (1') lo que uno hace cuando experimenta, entiende y juzga. Este paso es la experiencia interna, la consciencia, y ya lo ha ejercido todo mundo, aunque no es tan sencillo explicitar en qué consiste; para ello hay que dar los pasos más difíciles, comprender (2') la experiencia de uno al experimentar-entender-juzgar, y (3') juzgar si lo comprendido en (2') es correcto o no. La explicitación de estas operaciones, de su estructura, debe ayudar sobre todo a poner de manifiesto con claridad los criterios normativos inmanentes, llamados por Lonergan «preceptos trascendentales», que guían a cada nivel de consciencia, a saber: «Sé atento», «Sé inteligente», «Sé razonable», «Sé responsable».

Un segundo énfasis en la pedagogía del método empírico generalizado es reconocer que somos conscientes de diversas maneras. El flujo de nuestra consciencia se orienta hacia distintos intereses o fines (patterns of experience). El primero de ellos es el biológico, dominado por el interés de sobrevivir, evitar el peligro, buscar el alimento, fortalecer la propia vitalidad. Tenemos el patrón práctico o de sentido común, en el que nos ocupamos de solventar de la mejor manera los asuntos de la vida diaria. Está el dramático, en el que nuestra preocupación es el reconocimiento y aceptación de otros. Están los patrones de la gratuidad, que son poco frecuentes, pero muy importantes por su intensidad: el estético, el intelectual y el religioso. Es el patrón intelectual de experiencia, animado por el deseo puro de conocer, el protagonista central del Insight: Todos los patrones son parte de la riqueza de la vida humana y cada uno tiene su propio criterio normativo, pero la propuesta de Lonergan es que la teoría debe hacerse con la prioridad y los criterios del patrón intelectual; la filosofía debe confinarse a éste y ser fiel a sus exigencias normativas y, por ello, tiene que advertir la mezcla e interferencia de los criterios de un patrón en otro, que generan confusiones en el sujeto polimórfico. Uno de los principales conflictos se da entre el patrón biológico, que por su exigencia normativa de sobrevivir, opera con la noción de que lo real es lo que «está-ya-ahí-ahora-afuerarealmente». Este criterio va a chocar con el criterio del patrón intelectual de experiencia, para el cual las cosas reales no son los bultos 
que están ahí afuera, sino lo que es unidad-identidad-totalidad. Del patrón intelectual se desprenderá el criterio de que para llegar a conocer lo realmente real debemos aprender inteligentemente los datos de la experiencia y afirmarlos razonablemente. Lonergan señala desde la "Introducción" que algo crucial en la autoapropiación de la propia estructura dinámica cognoscitiva es descubrir que hay dos realismos: uno que opera en el sujeto de modo espontáneo y, por eso, se le puede llamar «ingenuo», muy cercano al materialismo; y otro, que pasa por el idealismo, pues descubre la centralidad de la consciencia empírica, inteligente y razonable, pero va más allá, y su descubrimiento pleno produce una extrañeza desconcertante (startling strangeness), una conversión del horizonte de lo real, pues ya no se trata ahora de echar un buen vistazo a lo que está ahí afuera, sino de ser fiel a la búsqueda de la verdad. El "incansable dinamismo veritativo" (Sánchez Leyva, 2013: 490) de Newman, así como el cambio que experimentó san Agustín al descubrir que «real» no significa ser un «cuerpo», son ejemplos privilegiados de dicha conversión intelectual.

En la pedagogía del Insight importa mucho comprender lo que es comprender -ocho largos capítulos, de los veinte del Insight, se destinan a tal fin- pues, por la influencia del patrón biológico, aunque entendamos correctamente, solemos pensar que entender es tener una especie de foto de lo que «está afuera». Sin embargo, entender no es conocer. Una teoría brillante, inteligente, no necesariamente es verdadera. Por eso siguen los capítulos nueve y diez, dedicados a que el lector trate de entender lo que hace cuando juzga si algo ya comprendido de hecho, lo es en efecto, o no; qué es lo que sucede en nosotros cuando asentimos a una proposición, cómo podemos saber si es verdad o no, si nunca podremos salirnos fuera de nosotros mismos a echar un buen vistazo a lo que está allá afuera: es aquí donde va a brillar como un faro lo que Lonergan leyó tan cuidadosamente de su mentor y guía.

Con todo, no basta con entender lo que es juzgar. Hay que juzgar si es verdad que uno conoce al experimentar, comprender y juzgar y a ello se dedican los capítulos centrales del Insight, que son el once, el doce y el trece, en los que se pide al lector que juzgue si en efecto 
conocer es aprender de manera inteligente los datos de la experiencia y afirmar razonablemente lo que uno ha entendido. Se trata de que el lector descubra en él mismo el sentido ilativo, la capacidad que existe en todo ser humano, y no sólo en el científico, o en el ilustrado, de llegar a la verdad, de aprender algo no de modo probable, o nocionalmente, sino de modo real, incondicionalmente.

La presencia inombrada del sentido ilativo de Newman

El capítulo diez investiga el fundamento inmediato del juicio y lo encuentra en la aprehensión del incondicionado virtual, una idea que fue precedida en mi pensamiento por cierta familiaridad con el sentido ilativo de Newman. Es diferente del realismo ingenuo y de la opinión empirista, la cual considera a la verificación como un asunto de atender a los datos, y no como una tarea de encontrar los datos que encajen con una hipótesis. Y difiere, además, por supuesto, de la vieja noción de que juzgar es un asunto de comparar conceptos y descubrir que uno implica al otro (Lonergan, 1974: 273). ${ }^{7}$

Los lonerganeanos estudiosos de la relación entre Lonergan y Newman han hecho magníficos trabajos reconstructivos de cómo llegó Lonergan a su noción del juicio a través de la $G A .{ }^{8}$ Lo extraño es

7 "Chapter ten investigates the immediate ground of judgment and find it in a grasp of the virtually unconditioned, a view that was preceded in my thinking by some acquaintance with Newman's illative sense. It differs from the naïve realist and empiricist opinion, which think of verification simply as a matter of attending to data and not as a matter of finding data that fit in with a hypothesis. It further differs, of course, from the old notion that judging can be a matter of comparing concepts and discovering that one entails another".

8 Debemos, desde luego, mencionar los trabajos pioneros de Frederick Crowe (1989). Está, también, todo el segundo capítulo, dedicado a Newman, de la biografía intelectual de la formación de Lonergan, que tan cuidadosamente realizó Richard M. Liddy (1993). El epígrafe de este libro es, incluso, la frase que Newman escogió para su tumba: "Ex umbris et imaginibus in veritatem". ("De las sombras y las imágenes, a la Verdad"). Está la biografía de William A. Mathews (2005), con más de 30 entradas en el nombre de Newman; especialmente, valen la pena los artículos de Worgul (1997) y Hammond 
que en el libro Insight no aparece el nombre de Newman. Es muy llamativo que entre los seis nombres que menciona en la "Introducción”, como hilos conductores de las piezas que el Insight pretende ensamblar en una sola perspectiva, no aparezca Newman. ${ }^{9}$ Lonergan dijo en una entrevista posterior que lo omitió porque debía de explicar qué dijo Newman y no quería hacer eso. Por ello algunos trabajos pioneros como el de George S. Worgul (1977) se ocuparon de mostrar el grado en el que se da esta influencia. Worgul nota muy agudamente que, desde el inicio del Insight, hay una velada pero muy fuerte referencia a la $G A .{ }^{10}$ En la "Introducción", Lonergan describe su trabajo como: «ensayo en ayuda de la autoapropiación» («essay in aid of self- appropriation»). El contexto en el que usa la expresión en la "Introducción" es para justificar que haya decidido comenzar con los ejemplos de intelecciones de la física y la matemática, por su ventaja psicológica de que en ellos uno entiende claramente cuando ha entendido. Explica cómo en el tema de la verdad el problema es más bien psicológico, y no tanto lógico, pues en la aprehensión de la verdad se involucra la totalidad de la persona: "He venido insistiendo en la seriedad de los motivos que me condujeron a comenzar este ensayo en ayuda de la autoapropiación por un examen de la física matemática” (Lonergan, 1999: 25).

En las notas editoriales a la versión del Insight en las Collected Works, Fred Crowe señala que el subtítulo del escrito que fue mecanografiado para enviarse a la editorial, rezaba: «Un ensayo para coadyuvar a la apropiación personal de nuestra propia autoconsciencia racional» («An Essay in Aid of Personal Appropriation of One's Own Rational Self-Consciousness»), pero que fue cambiado en el proceso de

(1989). Egan ya fue mencionado. Michael Paul Gallagher (2004) escribió un excelente artículo que, si bien no tiene un propósito propiamente «reconstructivo», aporta el estudio de los sermones universitarios en la obra de Lonergan. Como mencioné, mi propósito no es histórico; si tal fuera, tendría que ir a los Blandyke papers y analizar con mucho detalle el artículo de 1929 dedicado por completo a la GA.

9 Y sí Maréchal, con lo cual se favoreció la interpretación de Lonergan como un tomista trascendental. Lonergan menciona a Platón, Aristóteles, Tomás de Aquino, Descartes, Kant y Maréchal (Lonergan, 1999: 24-25).

10 Por ejemplo, en Worgul (1977: 318). 
publicación hasta llegar al subtítulo actual (Lonergan, 1992: 775), que hace pensar en el Ensayo sobre el entendimiento humano (An Essay Concerning Human Understanding) de John Locke. Pero, aunque el Essay de Locke está muy presente en Newman, lo está con importantes diferencias filosóficas, el título de la GA guarda un parecido con el de Locke, sobre todo en lo que toca al carácter retórico del mismo, ${ }^{11} \mathrm{de}$ modo que tenemos tres Essays relacionados, aunque en el de Lonergan finalmente acabara apareciendo la expresión «A Study».

En el epílogo, Lonergan vuelve a usar en dos ocasiones la misma expresión («An essay...»). En la primera, hablando de que el Insight se ha tratado de mantener en un plano estrictamente filosófico, dice que el último capítulo sobre el problema del mal pide que el punto de vista alcanzado en Insight se abra a un punto de vista superior, teológico, al cual el propio libro puede contribuir. Es interesante que la cita mencione la posible contribución a la apologética, lo cual lo acerca a la $G A$ que pretende mostrar la razonabilidad de la fe religiosa: ${ }^{12}$

11 Si bien la palabra Essay era común, Newman está respondiendo en buena medida, aunque no únicamente, ni como propósito central, al de Locke. Lo importante es que en ambos existe un interés por persuadir: "tanto Locke como Newman están operando en el amplio campo de la epistemología, pero para este ensayo, me concentraré en hacer una comparación de sus análisis de lo que yo llamo una «retórica del asentimiento», a saber, un análisis de cómo las personas son inducidas a dar su asentimiento a verdades proposicionales. Ni Locke ni Newman se propusieron escribir un texto retórico, pero dado que inducir el asentimiento en oyentes o en lectores es un objetivo primordial en todo discurso persuasivo, lo que estos dos hombres tienen que decir sobre el asentimiento debería interesar a todos los interesados en retórica" (Corbett, 1982: 40).

("Both Locke and Newman are operating in the broad arena of epistemology, but for this paper, I will focus on a comparison of their treatment of what I call a «rhetoric of assent»-that is, a treatment of how people are induced to give their assent to propositional truths. Neither Locke nor Newman set out to write a rhetoric text, but since the inducement of assent in listeners or readers is a primary objective of any persuasive discourse, what these two men have to say about assent should interest all rhetoricians").

12 Josep Vives, en su ensayo introductorio, escribe: "La Gramática del asentimiento es, fundamentalmente, un ensayo sobre la razonabilidad de la fe religiosa o, mejor dicho, de la certeza que tiene el cristiano sobre las verdades fundamentales del cristianismo. La obra de Newman ha de juzgarse por lo que pretende ser: su autor la tituló modestamente como "Ensayo», y así como antes de fijar la gramática de una lengua se requieren muchas contribuciones preliminares que analicen y den a conocer la naturaleza de los hechos lingüísticos, así Newman quiso darnos su contribución a lo que él concebía como una gramática o exposición sistemática de los hechos religiosos" (Newman, 2010: 12). 
Hemos venido preguntando si nuestro ensayo para coadyuvar a la apropiación personal de nuestra propia autoconsciencia racional puede poseer alguna significación para la teología, y hemos mencionado cierto número de contribuciones potenciales, aunque remotas, a la apologética y al método de la teología (Lonergan, 1999: 849, énfasis mío).

La segunda alusión, la encontramos en un contexto diferente. Lonergan presenta sus estudios sobre el verbum en Tomás de Aquino, como algo que está en estrecha relación con el Insight y señala que incluso podrían haber sido presentados en un solo libro. ${ }^{13}$

Por eso, mis investigaciones detalladas sobre el pensamiento del Aquinate acerca de la gratia operans y del verbum han continuado en el presente ensayo para coadyuvar a una apropiación personal de nuestra propia autoconsciencia racional. Sin duda, habría sido mejor si hubiese podido satisfacer en una sola obra a quienes buscan citas abundantes de santo Tomás y a quienes desean un sistema de pensamiento elaborado de manera independiente. Pero quizá me excusen quienes tienen suficiente energía para leer tanto mis estudios históricos como el presente libro, pues pienso que estarán de acuerdo en que cualquiera de estas tareas es de por sí bastante difícil y compleja (Lonergan, 1999: 854-855, énfasis mío).

En el Verbum (1997), se distingue el acto de intelección (insight), ${ }^{14}$ del acto de concebir, lo cual alude a la doctrina aristotélica de los dos

13 En los años cuarenta, Lonergan publicó cinco artículos sobre qué entendió santo Tomás por el verbum mentis, los cuales se publicarían después en forma de libro (Lonergan: 1997). Aunque con una pretensión hermenéutica, los artículos del Verbum (1997) le sirvieron a Lonergan como una base firme hacia su posición en el Insight. Por ejemplo, ahí aprehendió Lonergan de santo Tomás que conocemos por lo que somos y no por lo que hacemos. Ahí también aparece la idea de que es necesario moverse desde lo ganado por Aristóteles y santo Tomás a una postura más epistemológica en la que los significados no sean controlados primariamente por la metafísica.

14 Francisco Quijano, traductor del Insight, vertió «insight» como «acto de intelección»; sin embargo, en la portada se decidió dejar el término en inglés. Armando Bravo, quien ha traducido una gran variedad de textos de Lonergan, siguiendo a Eduardo Pérez Va- 
entendimientos. Para llegar a tal posición, Aristóteles y Santo Tomás debieron haber practicado el método empírico generalizado que en el Verbum es llamado «psicología introspectiva»; pero, como el control de los significados es ejercido desde la metafísica, dice Lonergan que ambos practicaron un método que no tematizaron.

Otro hallazgo del Verbum consiste en su distinción entre «comprender» $\mathrm{y}$ «juzgar», el segundo nivel de operaciones conscientes del tercero. Hay dos sentidos de «intelligere» en Santo Tomás, y el reflexivo no es una mera comparación de conceptos, como muchos manuales señalaban, sino algo muy cercano a lo que enseñó Newman: "En el presente capítulo la pretensión será que el intelligere del cual el juicio procede es un acto de entender reflexivo y crítico, no muy diferente del sentido ilativo de Newman" (Lonergan, 1997: 60). ${ }^{15}$ Permítaseme sugerir que, sin la GA, Lonergan no hubiera llegado a este capital descubrimiento hermenéutico.

La noción de «juicio» en el Insight

El capítulo nueve del Insight se llama "La noción del juicio". Es el capítulo más breve. En él se expone que experimentar, entender y juzgar forman una estructura. No se puede entender sin datos de experiencia y sin preguntas, del mismo modo que no se puede juzgar

lera, traduce «chispazo inteligente». Coloquialmente, decimos «Ya me cayó el veinte», «Ya se me prendió el foco». Newman describe el insight (que, por su parte, Vives traduce como «iluminación») que tuvo en Suiza, y que fue clave para escribir el libro que llevaba más de veinte años en su cabeza: “El hallazgo ocurrió mientras estaba de vacaciones en Suiza ese verano. Ahí, cerca del Lago de Ginebra, se topó con la idea: «Estás equivocado al comenzar desde la certidumbre -la certidumbre es solo una forma de asentimiento-; deberías comenzar contrastando asentimiento e inferencia». Sobre esto último hablé, considerándolo un punto clave en mis propias ideas" (Lash en Newman, 1992: 33).

("The breakthrough came while on holiday in Switzerland that summer. There, by the Lake of Geneva, he hit upon the idea: «You are wrong in beginning with certitude - certitude is only a kind of assent-you should begin with contrasting assent and inference». On that last I spoke, finding it a key to my own ideas").

15 "In the present chapter the contention will be that the intelligere from which the judgment proceeds is a reflective and critical act of understanding not unlike the act of Newman's illative sense". 
lo que no se ha entendido. Cada uno de estos niveles aporta algo propio: la experiencia nos proporciona los datos del conocimiento; los conceptos son la inteligibilidad de los datos, las relaciones que se aprehenden entre los datos; pero es en el juicio donde hay un incremento total en el conocimiento, pues sin el juicio tendríamos ideas, posibilidades, pero no aprehensión de lo real.

En el juicio estamos en el tercer nivel de operaciones conscientes. Así como pasamos del primero al segundo nivel preguntando «¿Qué?», «¿Por qué?», «¿Cómo?», «¿Quién?», pasamos al tercer nivel preguntando si eso que ya entendimos de verdad es así. Se procede, entonces, a ordenar y ponderar la evidencia, la operación más prolija de este tercer nivel. En algunos casos, se llega a juzgar si en efecto lo que se había comprendido es así o no.

Una idea del capítulo nueve muy cercana a Newman, es que el juicio es un compromiso personal. Yo no soy responsable de los datos de experiencia, pues son dados. Es verdad que puedo tratar de poner atención a ellos, pero no son propiamente mi responsabilidad. Tampoco lo soy respecto de las preguntas que surgen, ni mucho menos respecto de las intelecciones. La primera de cinco características de la descripción que se hace del insight, es que, de llegar, llega de repente e inesperadamente y sabemos, penosamente, que muchas veces más bien no llega. Tampoco soy responsable de la manera de conceptualizar lo que entendí, pues eso es parte de mi lenguaje, mi formación, mi herencia cultural. Pero de afirmar que algo es así, sí que soy responsable, pues perfectamente podría haber dicho: la verdad es que no sé, no tengo evidencia suficiente para afirmar eso y, sin embargo, nos dice Lonergan, citando a La Rochefoucauld: "Todo mundo se queja de su memoria, pero nadie de su juicio”. Escuchemos a Newman:

Por tanto, en lo que se refiere a raciocinios concretos, nos vemos en gran parte forzados a volver a aquella situación de la que la lógica prometía sacarnos. Hemos de juzgar por nosotros mismos, según nuestras propias luces y nuestros principios. Nuestro criterio de la verdad es, no tanto la manipulación de proposiciones, cuanto el carácter intelectual y moral de la persona que las mantiene y 
el efecto último y silencioso que sus argumentos o conclusiones ejercen sobre nuestra mente (Newman, 2010: 250, énfasis mío).

Otro punto en el que se advierte la influencia de Newman es sobre cierto aspecto paradójico del juicio, en tanto que cada juicio alude, necesariamente, a un contexto de juicios. Afirmar que escribo este texto en mi cubículo, está relacionado con los juicios de que existe algo que llamamos cubículo, el que afirma que presionar teclas es escribir, el que señala que existen las computadoras, etcétera. El juicio como incremento total se basa en nuestro conocimiento habitual, que no se va a hacer explícito en el momento de juzgar sobre algo específico; al contrario, si quisiéramos explicitarlo, nos sería imposible poder precisar el detalle.

Pero, además, nuestro conocimiento es dinámico en otro sentido. Es inevitablemente habitual. Pues no podemos hacer sino un solo juicio a la vez, y un solo juicio no puede hacer que todo lo que conocemos llegue a la luz plena del conocimiento actual. Un juicio puede ser muy comprehensivo y atestiguar así la profundidad y amplitud de nuestras perspectivas. Puede ser muy concreto y revelar así nuestra aprehensión de matices y detalles. Mas no puede ser a la vez comprehensivo y concreto. Todo lo que conocemos está de alguna manera en nosotros; está presente y es operativo en nuestro conocimiento; pero está oculto tras bambalinas, y no se revela sino en la exactitud con la cual se efectúa cada incremento menor en nuestro conocimiento (Lonergan, 1999: 342).

En su gran mayoría, ese conocimiento habitual está compuesto por creencias, es decir afirmaciones que aceptamos como verdaderas, perfectamente razonables, pero de las que no tenemos nosotros, por nosotros mismos, la evidencia de su verdad; pero lejos de ser éste un obstáculo para el conocimiento, por el contrario, es su condición de posibilidad. 


\section{El incondicionado virtual}

En el capítulo diez del Insight, "La comprensión refleja”, se profundiza en la comprensión de las operaciones que intervienen en el juicio y, además, se advierte que hay diferentes tipos de juicios, en gran medida porque hay diferentes contextos o campos semánticos. No es lo mismo la verdad de un juicio matemático que la de un juicio de la física o de uno de sentido común. Juzgar es afirmar que uno tiene evidencia para decir que algo es así. Sin la evidencia, estamos adivinando y no querer asentir cuando algo es evidente es una insensatez. El capítulo diez analiza, pues, cómo se pesa esa evidencia. ${ }^{16}$

Dijimos que la primera operación para juzgar es preguntarse si es verdad o no lo que uno entendió. Esto significa preguntar si uno tiene la evidencia para afirmar que lo comprendido («juicio prospectivo»), es o no algo incondicionado, o solo depende de que así lo queramos ver. Cuando nos preguntamos si es así o no, procedemos a reflexionar sobre nuestros datos de experiencia, nuestras preguntas orientadas a entender, nuestros conceptos y formulaciones. Ordenamos la evidencia y, sobre todo, la ponderamos. Es decir, nos preguntamos si tenemos todos los datos, si los observamos bien, si son pertinentes, si son coherentes las ideas que tenemos sobre ellos unas con otras, etcétera. La pregunta operante es qué condiciones tendría que satisfacer el prospecto de juicio para poder afirmar que es así, y no tan solo que así se me ocurrió. "Captar que la evidencia es suficiente para un juicio prospectivo es aprehender el juicio prospectivo como virtualmente incondicionado" (344). ${ }^{17}$ Un incondicionado virtual se distingue de uno formal, algo o alguien que de suyo no tuviera ninguna restricción ni condición en su modo de inteligir, lo que obviamente no es nuestro caso. Para nosotros, un juicio verdadero es un juicio que si bien tenía condiciones, se hizo

16 Lonergan señala la dificultad de explicitar exactamente lo que uno hace al aprehender la suficiencia de la evidencia de un juicio sobre el que estuvimos deliberando: "sin esfuerzos prolijos de análisis introspectivo, no podríamos decir qué ocurre exactamente en el acto de intelección refleja" (Lonergan, 1997: 343).

17 Todas las citas de los dos siguientes párrafos son de Lonergan (1999). 
incondicional por el proceso, por la fuerza (virtud) de satisfacer las condiciones: "Un incondicionado virtual comporta tres elementos, a saber: (1) un condicionado, (2) un nexo entre el condicionado y sus condiciones, y (3) el cumplimiento de las condiciones. Por tanto, un juicio prospectivo será virtualmente incondicionado, si (1) es el condicionado, (2) sus condiciones son conocidas y (3) las condiciones se cumplen" (344). Todo juicio verdadero es algo absoluto, incondicional. No es así "para mí". Es así, y punto. No era incondicional. Fue la comprensión refleja la que lo transformó de juicio prospectivo y condicionado "al estado de incondicionado virtual; y la comprensión refleja consuma esta transformación al aprehender las condiciones del condicionado y su cumplimiento" (344). ${ }^{18}$

La inferencia deductiva es un ejemplo de este esquema general de la comprensión refleja. En el modus ponens, decimos: Si A, entonces B; Es así que A; Luego, B. La premisa mayor vincula al condicionado B con sus condiciones A. La menor, afirma que se cumplieron las condiciones y, por tanto, que la conclusión es incondicionada. "La función, por tanto, de la forma de la inferencia deductiva es mostrar una conclusión como virtualmente incondicionada. El acto de intelección refleja aprehende la estructura y, por coerción racional, se sigue el juicio" (345, énfasis mío). Sin embargo, "la inferencia deductiva no puede ser el ejemplo básico del juicio, pues presupone que otros juicios son verdaderos" (345). ${ }^{19}$ Es sólo una ilustración.

18 Para una explicación de cómo plantea este tema Newman y del aparente conflicto entre afirmar que la mente humana es limitada por naturaleza y que sin embargo alcanza algo incondicionado, sin salirse de sí misma, puede consultarse a Rosario Athié (2001: 22).

19 Escuchemos ahora a Newman: “Está claro que la conclusión lógica formal no es el método por el que de hecho llegamos a obtener certeza acerca de lo concreto; y está claro también, después de lo que hemos apuntado, cuál es el método verdadero y necesario. Es la acumulación de probabilidades mutuamente independientes que resultan de la naturaleza y de las circunstancias del caso concreto de que se trata; probabilidades que son demasiado tenues para que puedan valer por separado, demasiado sutiles y tortuosas para que puedan convertirse en silogismos, y aun cuando fueran convertibles, demasiado numerosas y variadas para una tal conversión. Un dibujo difiere de un retrato de una persona en que éste no es una mera silueta continua, sino que contiene todos los detalles, las sombras y los colores armonizados: de la misma manera puede compararse el proceso multiforme e intrincado del raciocinio necesario 
La forma del acto mismo de intelección refleja es mucho más general que la forma de la inferencia deductiva. Si ha de haber una deducción, el nexo entre el condicionado y sus condiciones debe ser un juicio y el cumplimiento de las condiciones debe ser otro juicio. Ahora bien, los juicios son los productos finales del proceso cognoscitivo. Antes de que el nexo entre el condicionado y las condiciones se presentara en el acto de juicio, ya existía en un estado más rudimentario en el proceso cognoscitivo mismo. Antes de que el cumplimiento de las condiciones se hiciera patente en otro acto de juicio, ya estaba también presente en un estado más rudimentario en el proceso cognoscitivo. El hecho notable acerca del acto de intelección refleja es que puede valerse de esos elementos más rudimentarios del proceso cognoscitivo para alcanzar el incondicionado virtual (Lonergan, 1999: 345, énfasis mío).

Está hablando Newman más que Lonergan, o mejor dicho está hablando Newman con los términos de Lonergan. En el proceso cognoscitivo, está inscrito en forma rudimentaria la manera de establecer el nexo entre condicionado y condiciones, y la manera de saber si se alcanzaron éstas o no. Por eso comienza Lonergan su análisis con los juicios fácticos concretos: los juicios que el hombre de la calle ejerce desde siempre. El ejemplo que encontramos no es: «El Reino Unido es una isla», sino el hombre que regresa del trabajo a su hogar, encuentra las ventanas destrozadas, humo en el ambiente y charcos en el suelo, y entonces emite el juicio fáctico extremadamente limitado «Algo pasó». Lonergan muestra cómo hay un nexo entre las presentaciones sensibles al momento de llegar y las que recuerda al salir, cómo está operando en él la noción de «cosa» como algo que permanece en el tiempo, pero que, si presenta propiedades diferentes, es debido a un cambio. Sin embargo, los señalamientos del tipo anterior, son juicios, dice Lonergan, y "antes, empero, de ser o bien enunciados o bien juicios, existen como estructuras o

para llegar a los hechos concretos, con la operación ruda del tratamiento silogístico" (2010:239). 
procedimientos no analizados, inmanentes y ejercidos en el proceso cognoscitivo" (347). ${ }^{20}$ Una gran cantidad de presentaciones, de ideas, de situaciones, quedan integradas en el juicio: «Algo pasó». El ejemplo nos muestra que "el nexo entre el condicionado y las condiciones que han de cumplirse es una estructura inmanente y operativa en el proceso cognoscitivo. No es un juicio. No es un conjunto formulado de conceptos, como lo es una definición. Es sencillamente una manera de hacer las cosas, un procedimiento dentro del campo del conocimiento" (346).

Tales juicios fácticos se basan en intelecciones sobre situaciones concretas. «Intelección» porque, si bien suponen el procedimiento analítico de ordenar y ponderar la evidencia, hay un momento sintético en el que se aprehende que ya se alcanzó la suficiencia de la evidencia. Los insights forman conglomerados unos con otros, de tal modo que unos llevan a diversas preguntas relacionados con otros. De aquí surge una distinción con la que operamos espontáneamente: hay intelecciones vulnerables e invulnerables.

Las intelecciones son vulnerables cuando todavía quedan más preguntas por hacer sobre el mismo asunto. Pues las nuevas preguntas conducen a nuevas intelecciones, las cuales ciertamente complementan la intelección inicial, modifican en mayor o menor grado su expresión y sus implicaciones, y tal vez lleven a un parecer enteramente nuevo sobre el asunto. Pero, cuando ya no hay más preguntas, el acto de intelección es invulnerable. Pues sólo mediante nuevas preguntas es como surgen nuevas intelecciones, que completan, modifican o revisan el punto de vista y la explicación iniciales (Lonergan, 1999: 349).

Mas no se trata de que ya no haya preguntas ulteriores posibles, sino de que no haya preguntas ulteriores pertinentes posibles. Lonergan se sitúa en su epistemología entre la búsqueda de la certeza

20 "Antes de tal investigación y formulación, las estructuras y los procedimientos existen y son ejercidos; además, generalmente no operan mejor en virtud de que se haya efectuado el análisis" (Lonergan, 1999: 346). 
tipo Descartes, que pediría que ya no haya preguntas ulteriores posibles, y las diversas formas de escepticismo, que señalarían que siempre habrá preguntas ulteriores y nunca se llega a un fin. Tampoco se trata de que ya no se me ocurran preguntas ulteriores a mí, sino que ya no las haya. Por ello, se involucra la responsabilidad personal en el juicio, lo que podríamos denominar «la razonabilidad», la prudencia del justo medio, entre el que, a las primeras de cambio afirma que algo es así, y el indeciso que, a pesar de tener la evidencia para afirmar algo, se muestra vacilante. No puedo ahora entrar en mayores detalles epistemológicos, lo que quiero señalar es que Lonergan está siguiendo a Newman, al afirmar que el procedimiento cognoscitivo que opera es espontáneo, rudimentario si lo comparamos con la lógica formal; es auto-correctivo y, lo más importante: en muchas situaciones, llega a un fin. Tal vez sea en esta afirmación en la que se encuentra la influencia más profunda de Newman sobre Lonergan. Es decir, que somos capaces de hacer juicios verdaderos, que somos capaces de llegar a lo incondicionado, a lo absoluto. ${ }^{21}$

21 "La genialidad de Newman estuvo en su apelación a la experiencia concreta del pensamiento. Él rechazó la postura de que la lógica formal era la mejor expresión del conocimiento humano. Lonergan escribió: «La aseveración de Newman es que deberíamos estar satisfechos con el juicio sencillo porque no podemos analizar todos los fundamentos con los que hacemos el juicio. Si la expresión formal no hace justicia a nuestros fundamentos reales, la expresión formal no es el criterio natural de nuestros verdaderos fundamentos»" (CWL 20: 37). Sobre esta base, es posible tener certeza en asuntos de creencias aunque no podamos acomodar el juicio resultante en la forma de un silogismo. La mente humana reconoce «la convergencia de probabilidades» que hace que sea algo tonto dudar de los fundamentos de las propias creencias. Newman sostuvo que la creencia es un componente esencial del conocimiento humano porque hace posible la colaboración humana. Es más razonable confiar en el proceso de la creencia que dudar de todo. Lonergan escribió:“En lugar de declarar que no son dignos de confianza todos nuestros asentimientos por un nervioso miedo al error, debemos aceptarnos a nosotros mismos como somos, equivocados quizás en algunas opiniones pero en la mayor parte en lo correcto. Al nutrirse de estos puntos de vista y por la asimilación de los nuevos que vienen a la mente cuando ésta se desarrolla y crece en experiencia. El error es automáticamente dejado a un lado" (Lonergan, 2007: 37).

("Newman's brilliance was in his appeal to the concrete experience of thinking. He rejected the view that formal logic was the best expression of human knowledge. Lonergan writes: «Newman's contention is that we should be satisfied with the simple judgment because we cannot analyze all our ground for making the judgment. If formal expression does not justice our real grounds, the formal expression is not the natural criterion of our real grounds» (Lonergan, 2007: 37) On this account, it is possible 
No está de más aclarar que lo absoluto del juicio verdadero no significa que sea exhaustivo, en el sentido de que uno conociera todo de todo. El juicio, dice Lonergan, es un compromiso limitado, lo que significa que su validez es solo respecto a lo que se ha preguntado y se ha comprendido. Por supuesto que no basta con estos juicios fácticos; sin duda, revisten mucha importancia los juicios científicos, basados en generalizaciones, o en la búsqueda de leyes universales. Está, también, la complejidad de la verdad de los juicios matemáticos y de otras formas lógicas de los juicios, como las tautologías, pero todos ellos tienen como fundamento la estructura dinámica cognoscitiva que se ejerce en el hombre de la calle, en el «mixe»y en el scholar de Harvard. En el caso de Newman y Lonergan, es fundamental para su retórica que su lector (es decir, yo) aprenda esta racionalidad, esta coerción, esta fuerza racional como algo inherente en él (en mí), tan subjetivo como los deseos y los temores, y que descubra, por tanto, que la objetividad del conocimiento no solo no es algo contrapuesto a la subjetividad, sino que tiene a ésta, a la consciencia, como su fundamento.

La autoafirmación del sujeto cognoscente

El capítulo once del Insight le propone al lector que ejerza un juicio del cual puede estar absolutamente cierto. Es el juicio con sabor cartesiano que afirma yo soy un sujeto cognoscente. Es un juicio privilegiado, en el sentido de que en muchos juicios yo no dispongo de los datos relevantes. Pero, en el juicio que dice que soy un sujeto

to be certain in matters of belief even if we cannot arrange the resulting judgment into syllogistic form. The human mind recognizes «the convergences of probabilities» that makes it silly to doubt one's grounded beliefs. Newman held that belief is an essential component to human knowledge because it makes human collaboration possible. It makes more sense to trust the belief process than to doubt everything. Lonergan writes: «Instead of pronouncing all our assents untrustworthy from a nervous fear of error, we take ourselves as we find ourselves, wrong perhaps on a few opinions but the most part right. By the digestion of these views and by the assimilation of new ones which come to us as the mind develops and experience increases, error is automatically purged away (CWL20: 37)" (Schute, 2010: 32). 
cognoscente, el elemento clave son los datos de consciencia, por los que no solo experimento el rojo o lo frío, sino que experimento que soy una realidad consciente de manera empírica, inteligente y racional. El primer paso del método empírico generalizado explícito, ya todo mundo lo ha dado. La experiencia, sin embargo, no es conocimiento. Para afirmar que soy un sujeto cognoscente tengo que entender lo que es experimentar, entender y juzgar. Tengo que entender que, además, soy una unidad.

Lonergan ha dedicado diez capítulos, casi cuatrocientas páginas, a que el lector trate de entender los rasgos fundamentales de lo que es entender (capítulos uno al ocho) y juzgar (capítulos nueve y diez). Pero ahora el asunto es juzgar si es verdad que soy un sujeto cognoscente, deliberar si puedo realmente afirmar que de ese juicio he satisfecho las condiciones, y si tengo o no una intelección invulnerable sobre el hecho de que soy un sujeto cognoscente. Por supuesto, me doy cuenta de que ignoro muchos detalles de cómo se da la consciencia, cómo es que soy una unidad compleja que incluye propiedades físicas, químicas, celulares, psíquicas, intencionales, culturales. Pero no puedo afirmar que no soy una unidad sin contradecirme. No puedo afirmar que no tengo experiencia sin contradecirme. Es verdad que también de la comprensión ignoro cantidad de cosas, pero no puedo decir que no soy inteligente, ni racional sin caer en una contradicción «performativa»-perdón por el anglicismo- una contradicción entre lo que se afirma y lo que hace posible la realización de la afirmación.

Con todo y el sabor cartesiano que pueda tener este capítulo once, sería completamente equivocado leerlo así. Lonergan jamás ha pretendido buscar una proposición que le sirva de fundamento para su sistema. No está intentando responder la pregunta de si en verdad conocemos. El punto de partida ha sido el lector, tal como de hecho ya es, un sujeto cognoscente. La primera pregunta fue: «¿Qué es lo que tú, lector, haces cuando conoces?» La autoafirmación del sujeto cognoscente del capítulo once trata de mostrar al lector con un ejemplo real lo que el capítulo diez trataba de mostrar con el ejemplo ficticio del hombre que llega de trabajar para encontrar su casa desarreglada. El procedimiento se podría también ilustrar tipo Moore, diciendo: 
«Aquí está mi brazo derecho», o con otros juicios más complejos. Pero en la autoafirmación del sujeto cognoscente, los datos pertinentes me son perfectamente accesibles. Es un ejemplo privilegiado porque además muestra cómo este proceso dinámico está operando y por ello caería en una contradicción si lo quisiera negar.

Con todo, si bien la consciencia racional puede criticar el logro de la ciencia, no puede criticarse a sí misma. El espíritu crítico puede sopesar todo lo demás en la balanza, con la sola condición de no criticarse a sí mismo. Es una espontaneidad autoasertiva, que exige una razón suficiente para todo lo demás, pero no ofrece justificación de su exigencia. Surge, al modo de los hechos, para generar un conocimiento de los hechos, para impulsar el proceso cognoscitivo de las estructuras condicionadas del ejercicio de la inteligencia a la afirmación sin reservas del incondicionado. [...] Eso no es todo, pues yo estoy involucrado en ello, empeñado, comprometido. La disyuntiva entre la racionalidad y la irracionalidad es una alternativa abstracta, mas no una opción concreta. La racionalidad es mi propia dignidad, y estoy tan estrechamente apegado a ella que exigiría la mejor de las razones para abandonarla. Es más, estoy tan identificado con mi racionalidad que, cuando infrinjo sus normas superiores, me veo obligado o bien a arrepentirme de mi desmesura o bien a racionalizarla (Lonergan, 1999: 399-400).

El canadiense está señalando que mi subjetividad, con su inteligencia y racionalidad, es algo dado para mí. Permítaseme ahora hacer una larga cita de la $G A$, que es de mis favoritas y que encuentro muy cercana al texto lonerganeano anteriormente citado:

Si no puedo presuponer que yo existo, y que existo de una manera determinada, esto es, con una constitución mental determinada, no puedo especular sobre nada y valdría más que abandonara toda especulación. Tal como soy, esto es todo lo que soy. Éste es mi punto de vista esencial, y he de presuponerlo. De lo contrario, pensar no es más que un vano pasatiempo que no vale la pena. 
No hay un término medio entre usar mis facultades tal como son y arrojarme sobre el mundo exterior según el impulso ciego del momento, como la espuma sobre la cresta de las olas, para olvidarme simplemente de que existo. O soy lo que soy, o no soy nada. No puedo pensar, reflexionar o juzgar sin suponer el punto que querría precisamente probar. Mis ideas son siempre presuposiciones, y me muevo siempre en un círculo cerrado. No puedo evitar ser suficiente para mí mismo, porque no puedo hacer de mí otra cosa, y cambiarme sería destruirme a mí mismo. Si yo no hago uso de mí mismo, no tengo otro yo para hacer uso de él. Mi único objetivo es averiguar qué es lo que soy, para poder usar de ello. Para probar el valor y la autoridad de cualquiera de mis funciones, me basta con poder decir que me es connatural. Lo que he de averiguar son las leyes a las que vivo sujeto. La primera lección elemental de mis deberes es la resignación a las leyes de mi naturaleza, sean las que sean. Mi mayor desobediencia es la de impacientarme por lo que soy y entregarme a una aspiración ambiciosa por lo que no puedo ser, acariciar la desconfianza acerca de mis propias facultades y querer cambiar leyes que son idénticas a mí mismo (Newman, 2010: 283-284).

Lonergan consideró la autoafirmación del sujeto cognoscente como un juicio fáctico concreto, y de ahí trató de mostrar que negar que uno mismo es un sujeto cognoscente implica una contradicción performativa. El análisis de dicha contradicción muestra "los procedimientos naturales, inevitables y espontáneos que constituyen la posibilidad del conocimiento" (1999: 400), pero ello no se ha hecho a través de alguna forma de demostración del conocimiento, "sino pragmáticamente comprometiéndonos en el proceso" (1999: 400, énfasis mío). Y no hay en última instancia "un fundamento más profundo que ese compromiso pragmático” (1999: 400). No podemos, ni sería deseable buscar alcanzar una certeza absoluta tipo Descartes. La búsqueda del fundamento cognoscitivo implica un círculo vicioso, pues tal búsqueda supone el ejercicio del conocimiento, "y el fundamento por alcanzar no tendrá mejor garantía o más firmeza que la indagación ejercida para alcanzarlo" (1999: 400). 
Así como yo podría no existir, así como yo podría ser diferente de quien soy, así también mi conocimiento podría no existir y podría ser diferente de como es. El fundamento último de nuestro conocimiento no es una necesidad sino un hecho contingente, y el hecho no queda establecido antes de comprometernos en el conocimiento, sino de manera simultánea con este compromiso (Lonergan, 1999: 400).

El parecido de este texto con el párrafo que anteriormente cité de la $G A$ me parece asombroso. El talante es profundamente existencial, y tal vez hoy suene mejor que «existencial» el adjetivo «pragmático»; en efecto, es un compromiso pragmático. De hecho, soy éste; de hecho, soy racional y, de hecho, me interesa conocer. ${ }^{22}$ Lo que encontró Lonergan en la $G A$ no fue una doctrina filosófica, sino "algo que cuadraba con la manera en la que yo conocía las cosas" (Lonergan, 2004: 388). ${ }^{23}$ Una fundamentación absoluta del conocimiento

22 "La verdad que interesa más a Newman es la verdad concreta y, casi siempre, práctica. Es la verdad de los «hechos». La distinción central de su explicación se refiere a las proposiciones universales verdaderas, $\mathrm{y}$ a las proposiciones concretas $\mathrm{y}$ verdaderas. Las primeras son utilizadas en la inferencia, alcanzando la certeza; mientras que las segundas se acercan más al asentimiento. Para certificar si ha habido un asentimiento a una proposición real, Newman apela a la acción: su doctrina es eminentemente práctica" (Athié, 2001: 9).

23 En una larga entrevista que le hicieron a Lonergan, menciona lo siguiente de Newman: "Estaba buscando a alguien que tuviera sentido común, y supiera de lo que estaba hablando. ¿Y de qué estaba hablando Newman? Del juicio como asentimiento; de la aprehensión real y nocional, del asentimiento nocional y real. Estaba respondiendo al punto de vista liberal de que todos los juicios son más o menos probables pero ninguno es cierto. Y podía dar ejemplos. Caminando a lo largo de un camino puedes ver a un hombre debajo de un árbol, y te quedas sorprendido que él pueda estar ahí. Mientras más te aproximes, más cierto puedes estar que hay un hombre ahí. Está un poco oscuro. Estás muy, muy cerca, y pones tu mano y no hay nada ahí. ¿Estás equivocado esta segunda vez porque estuviste equivocado en la primera? ¡Newman te puede arrinconar con mucha astucia!" (Lonergan, Lambert et. al. 1982: 14).

(I was looking for someone who had some common sense, and knew what he was talking about. And what was Newman talking about? About judgment as assent; about real apprehension and notional apprehension, notional assent and real assent. He was answering the liberal view that all judgments are more or less probable but nothing is certain. And he could give examples. Walking along the road you see a man standing in a field underneath a tree, and you are surprised that he should be standing there. The closer you get the more certain you are that a man is standing there. It is a bit dark. You are really close and put out your hand and there's nothing there. Are you wrong 
no es un asunto humano, no hay fundamento "más profundo que ese compromiso pragmático". ${ }^{24}$ No se trata de excluir el error de modo absoluto, ${ }^{25}$ sino que se trata de permitir el proceso correctivo del autoaprendizaje al que nos lleva el deseo puro de conocer. ${ }^{26}$

the second time because you were wrong the first? He can work you into a corner very smartly!).

24 "Manteniendo firmemente como mantengo (con estos filósofos mencionados por último) la certeza de nuestro conocimiento, creo que es suficiente apelar a la voz común de la humanidad para probarla. Hemos de considerar como una facultad natural ordinaria lo que los hombres en general, de hecho, ejercitan. Ésta es una ley de nuestra inteligencia, que se cumple en toda clase de acciones, tanto si a priori hemos de considerarla como una ley como si no. El hecho de que en realidad esperamos es una prueba de que la esperanza como tal no es una extravagancia. Nuestra posesión de certezas es una prueba de que estar cierto no es una debilidad o un absurdo. No es de mi incumbencia aquí determinar cómo llegamos a la certeza: me basta con que sintamos de hecho que estamos ciertos. Esto es lo que, según creo, los escolásticos llaman tratar una cuestión in facto esse, en contraposición a tratarla in fieri. Si yo hubiese intentado esto último hubiera caído en la metafísica, mientras que mi finalidad es de tipo práctico, como la de Butler en su Analogy, aunque con una diferencia: Butler trata sobre la probabilidad, la duda, lo conveniente, el deber; mientras que en estas páginas yo me limitaré (aunque estoy muy lejos de excluir positivamente lo que se refiere al deber) a la verdad de las cosas y a la certeza que tiene nuestra mente sobre esta verdad" (Newman, 2011: 281-282).

25 "Newman nota en su análisis que «Estamos en un mundo de hechos, y hacemos uso de ellos» (GA: 346) Los hechos son cosas que aceptamos como reales y en las cuales nos encontramos naturalmente involucrados. El hecho de que estemos ocupados con las cosas que encontramos en el mundo es indicativo de nuestra confianza natural en nuestro mundo. Esta ingenua/natural postura de confianza en el mundo se acepta como fundamental, la duda es algo secundario" (Ono, 2011: 96).

(Newman notes in his analysis that «We are in a world of facts, and we use them» (GA:346). Facts are things that we accept as real and with which we find ourselves naturally involved. The fact that we engage with the things that we encounter in the world is indicative of a natural trust we have in our world. This natural/naïve stance of trust in the world is accepted as foundational-doubt is secondary).

26 "Leí muchas veces los principales pasajes teóricos de la Gramática del Asentimiento de Newman. La observación de Newman de que diez mil dificultades no constituyen una duda me ha colocado en una buena posición. Me ha animado a mirar las dificultades de frente, sin permitirles interferir con mi vocación o con mi fe. Su sentido ilativo llegó después a ser mi acto reflejo de comprensión" (Lonergan, 1974: 263).

(I read several times the more theoretical passages in Newman's A Grammar of Assent. Newman's remark that ten thousand difficulties do not make a doubt has served me in good stead. It encouraged me to look difficulties squarely in the eye, while not letting them interfere with my vocation or my faith. His illative sense later became my reflective act of understanding). 
Por eso es verdad lo mencionado por Aristóteles de que no hay un modo categórico de refutar a un escéptico. El escéptico, dice Lonergan, "no está involucrado en un conflicto con una necesidad absoluta. Podría no existir; podría no ser un sujeto cognoscente. La contradicción surge cuando utiliza el proceso cognoscitivo para negarlo" (1999: 400).

\section{Conclusión}

Suelo decir en varios escritos y en muchos foros que el gran problema cultural de nuestra época, es que vivimos en una cultura altamente diferenciada y mínimamente integrada y que tenemos una necesidad de integración, o de mediación, entre los distintos saberes, incluyendo el del sentido común, especialmente en el campo cognoscitivo, ético y político. La GA nos muestra que no hay una radical oposición entre el sentido común y el mundo de la teoría. No es verdad que solo exista el conocimiento en el campo científico. La creencia, lejos de ser algo irracional, es la que permite la colaboración entre los seres humanos. Por importantes que sean nuestras teorías generales, la prioridad la tiene el conocimiento de lo concreto, y la aprehensión real en la que el sujeto cognoscente queda explícitamente implicado. Newman fue algo así como un Descartes, ${ }^{27}$ en el sentido que es un autor que busca ir a explicitar los fundamentos, pero con sentido común. El peso de su indagación no estuvo en descartar el error y encontrar la certeza, sino en nuestra capacidad «natural» de conocer la verdad.

Bernard Lonergan fue transformado por el encuentro con Newman. John Henry Newman fue transformado por la lectura atenta de la tradición cristiana. Leer cuatro o cinco veces parece ser la única manera correcta de leer. Toda verdadera lectura nos permite ir más allá del encuentro con un tema, al encuentro con alguien, pero sobre todo al encuentro con nosotros mismos, con el misterio insondable que habita en nosotros y en el que habitamos.

27 Para situar esta afirmación, puede consultarse Ekeh, 2011. 


\section{Referencias bibliográficas}

Athié Lámbarri, R. (2001). El asentimiento en J. H. Newman. Pamplona: Servicio de Publicaciones de la Universidad de Navarra, Cuadernos del Anuario Filosófico, Serie Universitaria No. 141.

Corbett, EPJ. (1982). “A Comparison of John Locke and John Henry Newman on the Rhetoric of Assent”. En Rhetoric Review (1), 1: 40-49.

Crowe, FE. (1989). Appropriating the Lonergan idea. M. Vertin, ed. Washington DC: The Catholic University of America Press.

Egan, PA. (2007). "John Henry Newman and Bernard Lonergan: A Note on the Development of Christian Doctrine”. En Revista Portuguesa de Filosofia (63), 4: 1103 1123.

(1996). “Lonergan on Newman's Conversion”. En The Heythrop Journal, (37), 4: 437-455.

Ekeh, OP. (2011). “Newman's Cogito: John Henry Newman's Phenomenological Meditations on First Philosophy". En Heythrop Journal: A Bimonthly Review Of Philosophy And Theology (52), 1: 90-103.

Gallagher, MP. (2004). “Lonergan's Newman: Appropriated Affinities”. En Gregorianum (85), 4: 735-56

Hadot, P. (2006). Ejercicios espirituales y filosofía antigua. Madrid: Siruela.

Hammond, DM. (1989). "The Influence of Newman's Doctrine of Assent on the Thought of Bernard Lonergan”. En Method: Journal of Lonergan Studies (7), 2: 95-115.

Liddy, RM. (1993). Transforming Light: Intellectual Conversion in the Early Lonergan. Collegeville, Minn.: Liturgical Press.

Lonergan, B. (2007). Collected works of Bernard Lonergan. Vol. 20: Shorter Papers. RC Croken, HD Monsour y RM. Doran, eds. Toronto: University of Toronto Press.

. (2004). Collected works of Bernard Lonergan.Vol. 17: Philosophical and Theological Papers 1965-1980. RC Crocken y RM Doran, eds. Toronto: University of Toronto Press for Lonergan Research Institute of Regis College.

(1999). Insight: Estudio sobre la comprensión humana. F. Quijano, trad. Salamanca, México: Sígueme, Universidad Iberoamericana.

(1997). Collected works of Bernard Lonergan Vol. 2:Verbum:Word and Idea in Aquinas. FE Crowe y RM. Doran, eds. Toronto: University of Toronto Press.

(1988). Método en Teología. Traducción Gerardo Remolina. Salamanca: Sígueme.

(1992). Collected works of Bernard Lonergan Vol. 3: Insight: a Study of human understanding. Frederick E. Crowe y Robert M. Doran (eds.). Toronto: University of Toronto Press. 
(1974). A Second Collection: Papers by Bernard J.F. Lonergan S.J. W.F.J. Ryan y B.J. Tyrell (eds.). London, Philadelphia: Darton, Longman \& Todd , Westminster.

(1972). Method in Theology. New York, London: Herder and Herder, Darton, Longman \& Todd.

Lonergan, B, P. Lambert, et al. (1982). Caring about Meaning: Patterns in the life of Bernard Lonergan. P. Lambert, C. Tansey y C. Going, eds. Montreal, Quebec: Thomas More Institute Papers.

Mathews, William. (2005). Lonergan's Quest: A Study of Desire in the Authoring of Insight. Toronto, Buffalo, London: University of Toronto Press.

Newman, JH. (2010). Ensayo para contribuir a una gramática del asentimiento. J. Vives y LM Hernández, trad. Madrid: Encuentro.

. James Lash. 1992. An Essay in Aid of a Grammar of Assent. Notre Dame, Ind.: University of Notre Dame Press.

Sánchez Leyva, F. 2013. "El “illative sense” en la instancia veritativa según John Henry Newman” en Theologica Xaveriana, Vol. 63, n. 176, pp. 487-506.

Shute, M. 2010. Lonergan's Discovery of the Science of Economics. Toronto, Buffalo: University of Toronto Press.

Worgul, GS. (1977). “The Ghost of Newman in the Lonergan Corpus”. En Modern Schoolman (54), 4: 317-332. 